(C) 2021, The Authors. Published by Elsevier Inc. and Fass Inc. on behalf of the American Dairy Science Association ${ }^{\circledR}$. This is an open access article under the CC BY-NC-ND license (http://creativecommons.org/licenses/by-nc-nd/4.0/).

\title{
Integration of statistical inferences and machine learning algorithms for prediction of metritis cure in dairy cows
}

\author{
E. B. de Oliveira, ${ }^{1,2} \odot$ F. C. Ferreira, ${ }^{1,2} \odot$ K. N. Galvão, ${ }^{3,4} \odot$ J. Youn, ${ }^{5,6,7} \odot$ I. Tagkopoulos, ${ }^{5,6,7}$ N. Silva-del-Rio, ${ }^{1,2} \odot$ \\ R. V. V. Pereira, ${ }^{1} \odot$ V. S. Machado, ${ }^{8} \odot$ and F. S. Lima ${ }^{1 *} \oplus$ \\ ${ }^{1}$ Department of Population Health and Reproduction, School of Veterinary Medicine, University of California, Davis 95616 \\ ${ }^{2}$ Veterinary Medicine Teaching and Research Center, 18830 Road 112, Tulare, CA 93274 \\ ${ }^{3}$ Department of Large Animal Clinical Sciences, University of Florida, Gainesville 32610 \\ ${ }^{4}$ D. H. Barron Reproductive and Perinatal Biology Research Program, University of Florida, Gainesville 32610 \\ ${ }^{5}$ Department of Computer Science, University of California, Davis 95616 \\ ${ }^{6}$ Computer Science and Genome Center, University of California, Davis 95616 \\ ${ }^{7}$ AI Next Generation for Food System (AIFS), University of California, Davis 95616 \\ ${ }^{8}$ Department of Veterinary Sciences, College of Agricultural Sciences and Natural Resources, Texas Tech University, Lubbock 79409
}

\begin{abstract}
The study's objectives were to identify cow-level and environmental factors associated with metritis cure to predict metritis cure using traditional statistics and machine learning algorithms. The data set used was from a previous study comparing the efficacy of different therapies and self-cure for metritis. Metritis was defined as fetid, watery, reddish-brownish discharge, with or without fever. Cure was defined as an absence of metritis signs $12 \mathrm{~d}$ after diagnosis. Cows were randomly allocated to receive a subcutaneous injection of $6.6 \mathrm{mg} / \mathrm{kg}$ of ceftiofur crystalline-free acid (Excede, Zoetis) at the day of diagnosis and 3 d later $(\mathrm{n}=275)$; and no treatment at the time of metritis diagnosis (n $=275$ ). The variables days in milk (DIM) at metritis diagnosis, treatment, season of the metritis diagnosis, month of metritis diagnostic, number of lactation, parity, calving score, dystocia, retained fetal membranes, body condition score at d 5 postpartum, vulvovaginal laceration score, the rectal temperature at the metritis diagnosis, fever at diagnosis, milk production from the day before to metritis diagnosis, and milk production slope up to 5, 7, and 9 DIM were offered to univariate logistic regression. Variables included in the multivariable logistic regression model were selected from the univariate analysis according to $P$-value. Variables were offered to the model to assess the association between these factors and metritis cure. Additionally, the univariate logistic regression variables were offered to a recursive feature elimination to find the optimal subset of features for a machine learning algorithms
\end{abstract}

Received February 5, 2021.

Accepted July 23, 2021.

*Corresponding author: falima@ucdavis.edu analysis. Cows without vulvovaginal laceration had 1.91 higher odds of curing of metritis than cows with vulvovaginal laceration. Cows that developed metritis at $>7$ DIM had 2.09 higher odds of being cured than cows that developed metritis at $\leq 7$ DIM. For rectal temperature, each degree Celsius above $39.4^{\circ} \mathrm{C}$ led to lower odds to be cured than cows with rectal temperature $\leq 39.4^{\circ} \mathrm{C}$. Furthermore, milk production slope and milk production difference from the day before to the metritis diagnosis were essential variables to predict metritis cure. Cows that had reduced milk production from the day before to the metritis diagnosis had lower odds to be cured than cows with moderate milk production increase. The results from the multivariable logistic regression and receiver operating characteristic analysis indicated that cows developing metritis at $>7$ DIM, with increase in milk production, and with a rectal temperature $\leq 39.40^{\circ} \mathrm{C}$ had increased likelihood of cure of metritis with an accuracy of $75 \%$. The machine learning analysis showed that in addition to these variables, calving-related disorders, season, and month of metritis event were needed to predict whether the cow will cure or not from metritis with an accuracy $\geq 70 \%$ and F1 score (harmonic mean between precision and recall) $\geq 0.78$. Although machine learning algorithms are acknowledged as powerful tools for predictive classification, the current study was unable to replicate its potential benefits. More research is needed to optimize predictive models of metritis cure.

Key words: metritis cure, ceftiofur, dairy cow, machine learning

\section{INTRODUCTION}

Metritis, an acute inflammatory disease with systemic implications, affects 20 to $40 \%$ of the postpartum dairy 
cows in the first 21 DIM (LeBlanc et al., 2002; Sheldon et al., 2009; Dubuc et al., 2010). Risk factors such as dystocia, delivery of twin calves, vulva laceration, retained fetal membranes (RFM), or stillbirth can increase the range of metritis cases from 30 to $45 \%$ (Markusfeld, 1987; Benzaquen et al., 2007; Vieira-Neto et al., 2016). The consequences of metritis include reduced welfare, increased incidence of other diseases, decreased productive and reproductive performance, increased culling, and decreased profitability (Kossaibati and Esslemont, 1997; Sheldon et al., 2004; Stojkov et al., 2015).

In studies comparing cure-risk of treated and nontreated metritic cows, cure-risk of cows treated with ceftiofur ranges from 75 to $78 \%$; however, the cure-risk ranges from 55 to $62 \%$ for nontreated cows, a scenario that suggests that approximately 2 of 3 cows treated for metritis could recover without antibiotics (Chenault et al., 2004; McLaughlin et al., 2012; de Oliveira et al., 2020). The benefits of using ceftiofur extend beyond cure rates and include improved productive and reproductive performance and an estimated economic return of $\$ 207$ per cow (Silva et al., 2021). Third-generation cephalosporin drugs are essential in human and veterinary medicine. The threat of antimicrobial resistance has led the Center for Veterinary Medicine of the Food and Drug Administration to limit certain uses of cephalosporin to preserve antimicrobial effectiveness for human and veterinary medicine (FDA, 2020).

Reducing traditional antibiotics use is expected to delay the development of bacterial resistance to antibiotics (Fair and Tor, 2014). Results from a pilot study suggested that not treating metritis cases without fever occurring within the first 5 DIM had no negative outcomes (Sannmann et al., 2013). The development of novel strategies that identify cows with metritis that undergo cure may reduce antibiotic use while also providing economic and sustainability benefits for farmers. Developing models to predict cure based on cow-level and environmental risk factors may help identify cows during the postpartum period with metritis that may not need or benefit from the antibiotic.

An alternative approach to develop predictive models in a large data set such as in agriculture-related studies is machine learning algorithms (Grzesiak et al., 2010). The lack of restrictive parametric assumptions for data analysis and the development of predictive tools makes machine learning algorithms a good candidate for improving the prediction of classic statistical models (Grzesiak et al., 2010). Studies using machine learning have been used to predict cow performance or disease events using large and complex data sets (Shahinfar et al., 2012; Hempstalk et al., 2015). Studies indicate that risk factors for metritis and environmental fac- tors could increase the chance of treatment failure (de Oliveira et al., 2020; Machado et al., 2020). However, there is limited information showing whether or not factors influencing metritis cure in treated and nontreated cows are similar. The current study offers a unique platform to evaluate what factors contribute to the success of metritis cure in a randomized experiment with metritic cows receiving or not receiving conventional antimicrobials.

The first objective of this study was to identify cowlevel and environmental factors associated with the likelihood of metritis cure. Our second objective was to evaluate the performance of traditional statistical models and a machine learning algorithm to predict metritis cure. Our premise was that cow and environmental-related factors could predict metritis cure with traditional statistical models and machine learning algorithms.

\section{MATERIALS AND METHODS}

\section{Data and Animals}

Our data set was from a previous study that compared the efficacy of different therapies and metritis cure (de Oliveira et al., 2020). Briefly, the study was conducted in 3 dairies located in North Central Florida from May 2016 to June 2017. Cows were diagnosed for metritis at 5, 7, and 9 DIM using the Metricheck device (Simcro). Cows with fetid, watery, and reddishbrownish discharge, with or without pieces of necrotic tissue present, were classified as having metritis (Sheldon et al., 2006). Cows diagnosed with metritis were blocked by parity (primiparous or multiparous) and, within each block, were randomly assigned to 1 of 3 treatments as follows: (1) chitosan microparticles ( $\mathrm{n}=$ 276) intrauterine infusion of $24 \mathrm{~g}$ of chitosan microparticles at diagnosis and 2 and 4 d later; (2) subcutaneous injections of $6.6 \mathrm{mg} / \mathrm{kg}$ of BW of ceftiofur crystallinefree acid (Excede, Zoetis) in the base of the ear at the day of diagnosis and $3 \mathrm{~d}$ later $(\mathbf{C E F} ; \mathrm{n}=275)$; and (3) no treatment at the time of metritis diagnosis $(\mathbf{C O N}$; $\mathrm{n}=275$ ). Because chitosan microparticles was not an efficacious therapy for metritis, only cows enrolled in the CEF or CON groups were included in the present study $(\mathrm{n}=550)$. At 5 DIM, all cows were scored for body condition $(1=$ thin, $5=$ obese; Ferguson et al., 1994) and vulvovaginal laceration score (VLS; $0=$ no laceration; $1=$ laceration $<2 \mathrm{~cm}$ at dorsal commissure or internal vaginal wall; $2=$ laceration $>2 \mathrm{~cm}$; Vieira-Neto et al., 2016). The vulvovaginal laceration was categorized (VLC) into "no" $(<2 \mathrm{~cm})$ or "yes" $(\geq 2$ $\mathrm{cm})$. Rectal temperature was measured at enrollment, 
and cows with a rectal temperature $\geq 39.5^{\circ} \mathrm{C}$ were considered febrile. Additionally, the occurrence of dystocia, twins, RFM, and stillbirth were recorded. The criterion to determine cure was an absence of the characteristic fetid, watery, reddish-brownish discharge at $12 \mathrm{~d}$ after enrollment. A cow was considered a treatment failure if she was sold or died before d 12 after metritis diagnosis.

Cows that presented severe dehydration, anorexia, weakness, severe depression, systemic shock, or any other clinical signs attributable to metritis (other than characteristic vaginal discharge and fever) received antibiotic therapy according to the farm protocol and were considered a treatment failure. Milk yield, number of services, average DIM when cows left the herd, reasons for culling, and mortality of the cows enrolled in the study were also recorded. Additional diseases were not accounted in the analysis.

\section{Statistical Analysis}

From the data collected in the study described above, we defined, categorized, and used the following variables for every cow enrolled in the study: the DIM on $d$ 0 (DIM D0), categorical days in milk at metritis diagnosis (metritis diagnosed at the first week postpartum vs. diagnosed after the first week postpartum), treatment ( $\mathrm{CEF}$ vs. CON), season of the metritis diagnosis [cool (September-May) vs. hot (June-August)], month of metritis diagnosis (January-December), lactation number, parity (primiparous vs. multiparous), calving score $(1=$ unassisted, 2 = easy pull or slight problem, $3=$ moderate pull, and 4 hard pull), dystocia [no (calving score $=1$ ) vs. yes (calving score >1)], RFM (yes vs. no), BCS at d 5 postpartum (BCS5), categorical BCS5 (low $\leq 3.0$, moderate $>3.0$ or $<3.75$, or high $\geq 3.75$ ), VLS $(0,1$, or 2$)$, categorical VLS (VLC, $>2$ $\mathrm{cm}$ vs. $<2 \mathrm{~cm})$, rectal temperature at the metritis diagnosis, fever at the metritis diagnosis $\left[\right.$ yes $\left(\geq 39.5^{\circ} \mathrm{C}\right)$ vs. no $\left.\left(\leq 39.5^{\circ} \mathrm{C}\right)\right]$, milk production from the day before diagnosis to the metritis diagnosis continuous (Vmilk), milk production from the day before to the metritis diagnosis categorical (Milk Var), and the slope of milk production up to 5, 7, and 9 DIM. The slopes of milk production up to 5, 7, and 9 DIM were calculated using linear regression models (PROC REG, SAS 9.4; SAS Institute Inc.). The model included daily milk production $(\mathrm{kg})$ as a dependent variable and DIM as an independent variable. Milk increase was categorized based on the percentile of milk production increased obtained from the linear regression $($ low $=\mathrm{P} 1-\mathrm{P} 25$; moderate $=$ $\mathrm{P} 25-\mathrm{P} 75$, or high $>\mathrm{P} 75$, where $\mathrm{P}=$ percentile).

Descriptive statistical analysis regarding continuous data, such as DIM D0, the slope of milk production, milk difference, the rectal temperature at enrollment, and BCS at metritis diagnosis, were compared nonparametrically using the PROC NPAR1WAY of SAS, and $P$-values from the Kruskal-Wallis test are reported. Mean values for normally distributed continuous variables were generated using ANOVA to compare noncured and cured groups. For the categorical variables (dystocia, parity, RFM, VLC), GLIMMIX procedure of SAS was used to compare cows that cured or failed to cure metritis, and PROC FREQ of SAS was used to report proportions.

A 3-step approach was used to select the variables to be included in the final multivariate model. First, univariable analyses for each independent variable were performed to assess their association with the odds of metritis cure with farm as a random effect (de Oliveira et al., 2020), and variables were ranked according to their $P$-value. Second, multivariable analyses were performed using PROC MIXED and GLIMMIX with maximum likelihood and Laplace parameter estimation methods, respectively. All variables were offered to the model according to their $P$-value from the univariate analysis (smallest to largest), and the model Akaike information criterion (AIC) was used to select the final model variables (stepwise elimination approach). The model with the lowest AIC was used. Finally, PROC MIXED and GLIMMIX were used with REML and residual subject-specific pseudolikelihood default specifications for parameter estimation. The final model for the multivariate analysis included cure as a dependent variable and the effects of treatment, vulvovaginal laceration, week postpartum of metritis, rectal temperature at metritis diagnosis, and milk difference as an independent variable. Furthermore, first-order interactions for all variables were added to the model, and significant ones were kept.

The multivariable analysis results are reported as the ratio of the odds of metritis cure for each risk factor. The unit of analysis was the cow. Statistical significance was declared for all models described above if $P$ $\leq 0.05$, and a tendency was considered if $0.05<P$ $<0.10$. For variables that had $P<0.05$ in the multivariate logistic regression models, receiver operating characteristic (ROC) curves were performed using MedCalc (MedCalc Software, ver. 18.11.6) to determine the most accurate threshold to predict metritis cure. A multivariable ROC curves analysis was performed using the logistic procedure of SAS. The multivariable ROC included the variables that were significant in the multivariable logistic regression model. The results of ROC were reported as sensitivity (Se), specificity (Sp), positive predictive value (PPV), and negative predictive value (NPV) and were considered as follows: less 
than 0.2 as low, 0.2 to 0.4 as fair, 0.4 to 0.6 as moderate, 0.6 to 0.8 as substantial, and above 0.8 as high (Dohoo et al., 2009).

\section{Machine Learning Algorithms}

Data Preprocessing. Machine learning algorithms were used to predict cows that would cure or not once diagnosed with metritis. The data set contained instances (observations) of 550 cows described by 24 attributes (variables), where 22 were independent variables, and 2 were dependent variables (Figure 1; De Oliveira et al., 2021). Among the 2 dependent variables, the target variable "cured" was a binary variable that described whether the animal cured up to $12 \mathrm{~d}$ after metritis diagnosis. As for the 22 independent variables, we applied standard data preprocessing pipeline of feature scaling (FS), missing value imputation (MVI), and outlier detection (OD) in the specified order using the scikitlearn Python package and missingpy Python library (https://github.com/epsilon-machine/missingpy). We tested 3 variations of FS (standard, minmax, and robust), 3 variations of MVI [k-nearest neighbors (KNN; Troyanskaya et al., 2001), multivariate imputation by chained equation (MICE; van Buuren and Karin, 2010), and miss forest (Stekhoven and Bühlmann, 2012)], and 2 variations of OD [isolation forest (Liu et al., 2008), and local outlier factor (LOF; Breunig et al., 2000)].

Feature Selection. To find the optimal subset of features for the classifier, we performed recursive feature elimination on the preprocessed data using the Python library Mlxtend (https://github.com/rasbt/mlxtend). For each step of recursive feature elimination, we removed a single feature associated with the best classifier performance if it was removed until only a single feature (most informative) remained. For selecting the optimal subset, we chose the smallest feature subset that was within 1 standard error of the cross-validation performance instead of the feature subset with the best cross-validation performance (described below). This parsimonious feature selection helped reduce the number of features as much as possible while retaining a reasonable performance, thus reducing the cost of data collection, reducing the training time, and allowing for generalizability.

Model Selection Pipeline. The overall model selection pipeline included the following steps: data preprocessing, feature selection, up-sampling using SMOTE (Valdés et al., 2018), and classifier training. For up-sampling, we resampled only the minority class (not cured) to match the number of the majority class (cured). Therefore, the final number of samples used for training the machine learning algorithms was 794
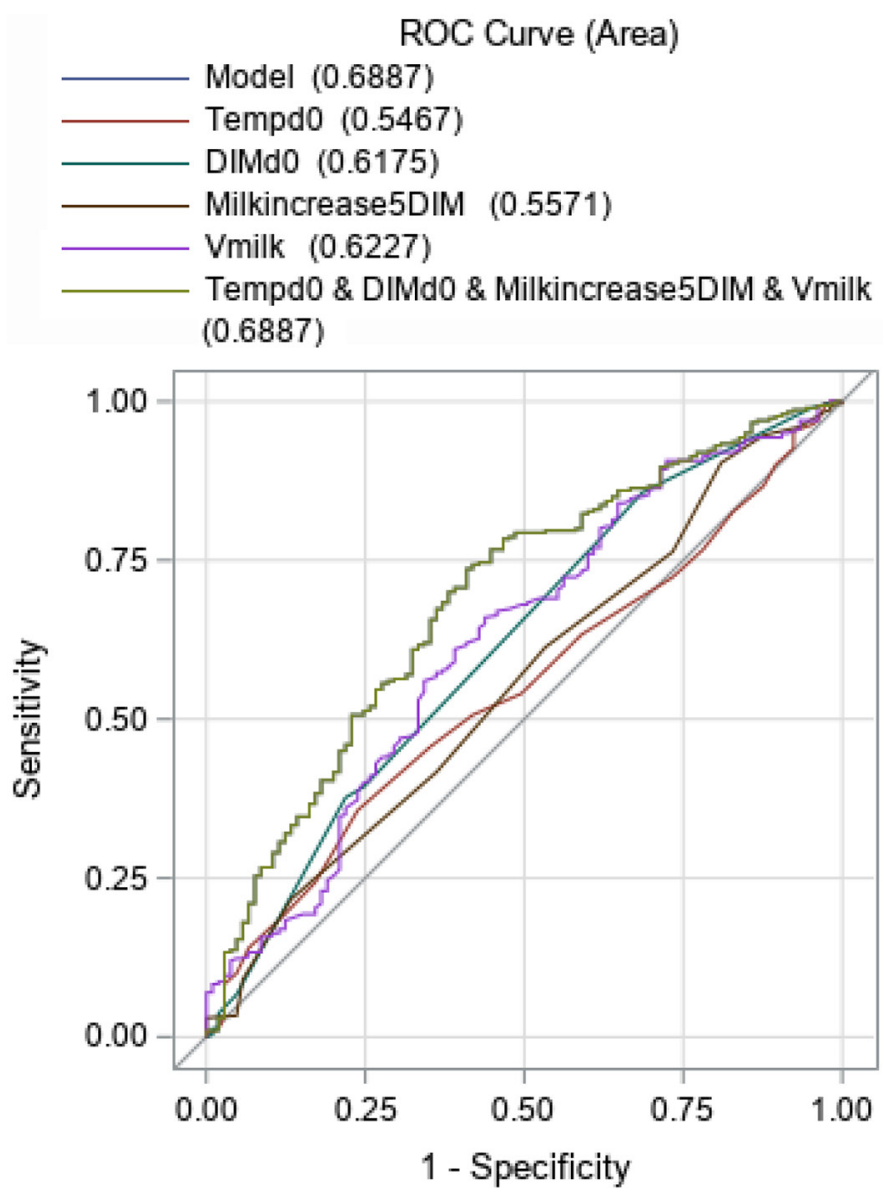

Figure 1. Comparison of receiver operating characteristic (ROC) curves with optimal threshold values for the whole model. Tempd0 $=$ rectal temperature at metritis diagnosis; DIMd0 $=$ DIM at metritis diagnosis; Milkincrease5DIM = slope of milk increase up to 5 DIM; Vmilk $=$ milk production difference from day before to diagnosis.

(397 from each class). Up-sampling was only applied to the training process. We trained 7 classifiers of varying complexity [decision tree (Mitchell, 1997), Gaussian naïve Bayes (Witten et al., 2011), multinomial naïve Bayes (Domingos and Pazzani, 1997), SVC (Hu et al., 2016a,b), AdaBoost (Morra et al., 2010), random forest (Ho, 1995; Breiman, 2001), and artificial neural network (Zafeiris et al., 2018)]. Therefore, the model selection pipeline had 126 possible combinations (3 FS, 3 MVI, 2 OD, and 7 classifiers). We then performed a grid search to find the best hyperparameter of the classifier for that given combination. For evaluation, we performed 5 -fold cross-validation, which consisted of partitioning the data into 5 equally or approximately equally sized segments. Subsequently, 5 iterations of training and validation were performed such that within each iteration, a different segment of the data was used as testing data, whereas the other 4 segments were used 
for training the data. The classification performance measured the classifier accuracy, Se, Sp, PPV, NPV, precision, and harmonic mean between precision and recall (F1 score). Accuracy was calculated as the ratio between the sum of the true positive (TP) and true negative (TN) results and the sum of $\mathrm{TP}, \mathrm{TN}$, false positives (FP), and false negatives. Precision was calculated as the ratio between TP and the sum of TP and $\mathrm{FP}$. The false detection rate was calculated as the ratio between FP and the sum of FP and TP. The F1 score was calculated using the formula $(2 \times \mathrm{Se} \times$ precision $) /$ (Se + precision). The performance of the classifier was optimized using the F1 score.

Recommender System. A recommender system is a personalized recommendation to handle information overload problems and improve description of an outcome of interest (Lu et al., 2015). All results presented in the manuscript were based on the recommender system. Using the best model among the 126 combinations from the model selection pipeline, we built a recommender system that recommended which cows would cure or not from metritis. We recommended setting the threshold $\theta$ to the difference in cured and not-cured cows' prior probabilities.

Experimental Scenarios. We trained the machine learning models on 4 different subsets of the features to analyze different end-user case scenarios regarding data availability on individual cow daily milk production (Table 1). In scenario 1, we assumed that milk production data were not available, but all other features were used. Scenario 2 had all fixed features and milk production slope up to 5, 7, and 9 DIM features. Scenario 3 had all fixed features and milk differences from the day before to metritis diagnosis categorical (MilkVar) and continuous (Vmilk) features. Scenario 4 had all fixed and milk production features.

\section{RESULTS}

\section{Descriptive Statistics}

The descriptive statistics regarding the number of cows cured, parity, dystocia, RFM, VLS, mean BCS5, mean DIM D0, the rectal temperature at enrollment, mean of milk production slope at 5 DIM, and mean of milk difference from the day before to the metritis diagnosis for cows cured and noncured are presented in Table 2. The proportion of cows with vulvovaginal laceration was greater $(P<0.01)$ in the noncured than in the cured group. Mean DIM D0 and milk production slope at 5 DIM were greater $(P<0.01)$ in cured than in the noncured cows. The milk difference from the day before diagnosis to the metritis diagnosis was different $(P<0.01)$ between the groups, being positive for

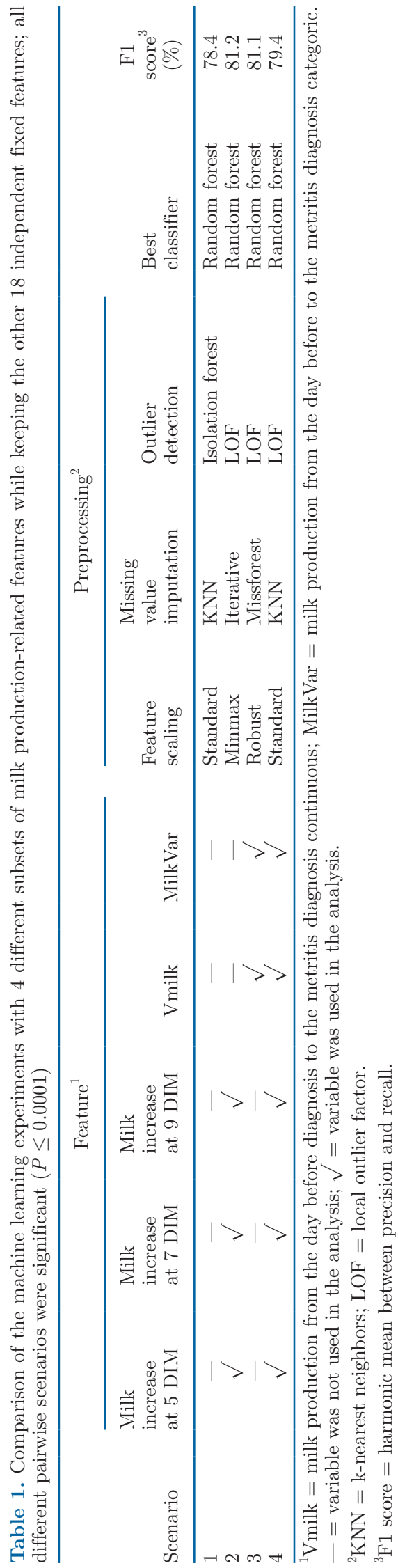


Table 2. Descriptive statistics of cured and noncured groups used in logistic regression and machine learning analysis

\begin{tabular}{|c|c|c|c|}
\hline \multirow[b]{2}{*}{ Item } & \multicolumn{2}{|c|}{ Cured } & \multirow[b]{2}{*}{$P$-value } \\
\hline & Yes & No & \\
\hline Animals enrolled [no. (\%)] & $397(72.2)$ & $153(27.8)$ & \\
\hline Primiparous animals enrolled [no. (\%)] & $207(72.4)$ & $79(27.6)$ & 0.60 \\
\hline Multiparous animals enrolled [no. (\%)] & $190(72.0)$ & $74(28.0)$ & \\
\hline Dystocia $[$ no. $(\%)]$ & $112(28.2)$ & $55(35.9)$ & 0.18 \\
\hline Animals with retained placenta [no. (\%)] & $164(41.3)$ & $65(42.5)$ & 0.46 \\
\hline Animals with vulvovaginal laceration [no. (\%)] & $90(22.7)$ & $56(36.6)$ & $<0.01$ \\
\hline Mean milk production slope at $5 \mathrm{DIM}^{1}$ & 2.07 & 1.38 & $<0.01$ \\
\hline Mean milk difference $(\mathrm{kg})^{2}$ & 1.29 & -1.50 & $<0.01$ \\
\hline Mean BCS at 5 DIM & 3.43 & 3.42 & 0.88 \\
\hline Mean DIM at metritis diagnosis & 7.6 & 6.9 & $<0.01$ \\
\hline Mean rectal temperature at enrollment $\left({ }^{\circ} \mathrm{C}\right)$ & 39.3 & 39.5 & 0.02 \\
\hline
\end{tabular}

cured cows and negative for noncured cows (Table 2). The rectal temperature at enrollment was lower $(P=$ 0.02 ) in cured cows than the noncured herdmates. The remained variables analyzed were not different between cured and noncured cows (Table 2).

\section{Association of Cow-Level and Environmental Factors with Metritis Cure}

The outcomes of the initial univariable logistic regression models evaluating the association of categorical variables with metritis cure is presented in Table 3. Week of enrollment $(P<0.03)$, treatment $(P<$ $0.01)$, VLC $(P<0.01)$, VLS $(P<0.01)$, slope of milk production up to 5 DIM categorical $(P=0.02)$, and categorical milk difference from day before diagnosis to metritis diagnosis $(P<0.01)$ were positively associated with metritis cure. Season $(P=0.12)$, month of enrollment $(P=0.18)$, parity $(P=0.60)$, dystocia $(P=$ $0.18), \operatorname{RFM}(P=0.46), \operatorname{BCSC}(P=0.85)$, and fever $(P$ $=0.16)$ were not associated with metritis cure. For the continuous variable, DIM at enrollment $(P<0.01)$, rectal temperature at enrollment $(P=0.02)$, slope of milk production up to 5 DIM $(P=0.01)$, and milk difference from day before diagnosis to metritis diagnosis $(P$ $<0.01$ ) were positively associated with metritis cure, whereas lactation number $(P=0.31)$ and BCS5 $(P=$ 0.60 ) were not associated with metritis cure (Table 4 ).

The outcomes from the final multivariable logistic regression model evaluating the association between cow-related factors and metritis cure are presented in Table 5. Treatment affected $(P<0.01)$ the odds of metritis cure, with CEF having 1.99 higher odds of cure than cows in CON group. Cows without vulvovaginal laceration had 1.91 higher odds of cure than cows with vulvovaginal laceration $(P<0.01)$. There was a tendency for an interaction between treatment and vulvovaginal laceration $(P=0.06)$. Cows without vulva laceration treated with ceftiofur had 3.28 higher odds of cure than cows with vulva laceration. In contrast, cows with vulvovaginal laceration treated with ceftiofur had no differences in cure when compared with control cows. Cows that developed metritis after the first week postpartum had 2.09 higher $(P=0.05)$ odds of being cured than cows that developed metritis in the first week postpartum. For rectal temperature, each degree Celsius above $39.4^{\circ} \mathrm{C}$ led to a tendency $(P=0.08)$ of 0.68 lower odds to be cured than cows with rectal temperature $\leq 39.4^{\circ} \mathrm{C}$. Cows that had reduced milk production from the day before diagnosis to the metritis diagnosis had 0.46 lower odds to be cured than cows with moderate milk production increase $(P<0.01)$.

The multivariable ROC curve revealed an area under the curve (AUC) for the model of 0.69 (Figure 1). The AUC in the multivariable ROC for rectal temperature at d 0, DIM D0, milk difference, and milk production slope 5 DIM were $0.55,0.62,0.62$, and 0.56 , respectively. The Se, Sp, PPV, and NPV for the multivariable ROC were, $0.98,0.08,0.75$, and 0.08 , respectively. The accuracy, precision, and F1 for the multivariable ROC were, $0.75,0.75$, and 0.85 , respectively.

Optimal thresholds for rectal temperature, DIM D0, milk difference from the day before to the metritis diagnosis, and slope of milk production up to 5 DIM to predict cure were determined using univariate ROC curves (Figure 2). Among cows that cured, the milk difference threshold that maximized the AUC (AUC = $0.61)$ was $>0.09 \mathrm{~kg}(\%$ cows below threshold $=60 \%)$, the threshold was $>5 \mathrm{~d}$ ( $\%$ cows below threshold $=$ $21.6 \%)$ for DIM ( $\mathrm{AUC}=0.62)$, and the temperature cut point for metritis cure $(\mathrm{AUC}=0.56)$ was $\leq 39.2^{\circ} \mathrm{C}$ (\% cows below threshold $=42 \%)$. 
Table 3. Univariate logistic regression model evaluating the association of several categoric variables with metritis cure

\begin{tabular}{|c|c|c|c|}
\hline Variable $^{1}$ & Level & $\begin{array}{l}\text { Odds ratio } \\
(95 \% \mathrm{CI})\end{array}$ & $P$-value \\
\hline $\mathrm{BCSC}$ & $\begin{array}{l}\text { Low } \\
\text { Moderate } \\
\text { High }\end{array}$ & $\begin{array}{c}0.87(0.48-1.59) \\
\text { Baseline } \\
1.06(0.66-1.70)\end{array}$ & 0.85 \\
\hline Dystocia & $\begin{array}{l}\text { Yes } \\
\text { No }\end{array}$ & $\begin{array}{c}\text { Baseline } \\
1.31(0.88-1.94)\end{array}$ & 0.18 \\
\hline Fever & $\begin{array}{l}\text { Yes } \\
\text { No }\end{array}$ & $\begin{array}{c}\text { Baseline } \\
1.30(0.89-1.89)\end{array}$ & 0.16 \\
\hline Milk difference & $\begin{array}{l}\text { Negative } \\
\text { Moderate } \\
\text { High }\end{array}$ & $\begin{array}{c}0.41(0.2-0.70) \\
\text { Baseline } \\
0.84(0.45-1.57)\end{array}$ & $<0.01$ \\
\hline Milk production slope 5 DIM & $\begin{array}{l}\text { Low } \\
\text { Moderate } \\
\text { High }\end{array}$ & $\begin{array}{c}0.78(0.49-1.23) \\
\text { Baseline } \\
1.88(0.99-3.60)\end{array}$ & 0.02 \\
\hline Parity & $\begin{array}{l}\text { Primiparous } \\
\text { Multiparous }\end{array}$ & $\begin{array}{c}\text { Baseline } \\
1.11(0.74-1.66)\end{array}$ & 0.60 \\
\hline RFM & $\begin{array}{l}\text { Yes } \\
\text { No }\end{array}$ & $\begin{array}{c}\text { Baseline } \\
1.15(0.78-1.71)\end{array}$ & 0.46 \\
\hline Season & $\begin{array}{l}\text { Hot } \\
\text { Cool }\end{array}$ & $\begin{array}{c}\text { Baseline } \\
0.67(0.41-1.12)\end{array}$ & 0.12 \\
\hline Treatment & $\begin{array}{l}\text { Control } \\
\text { Ceftiofur }\end{array}$ & $\begin{array}{c}\text { Baseline } \\
2.11(1.44-3.11)\end{array}$ & $<0.01$ \\
\hline VLC & $\begin{array}{l}\text { Yes } \\
\text { No }\end{array}$ & $\begin{array}{c}\text { Baseline } \\
2.13(1.41-3.22)\end{array}$ & $<0.01$ \\
\hline VLS & $\begin{array}{l}\text { Score } 0 \\
\text { Score } 1 \\
\text { Score } 2\end{array}$ & $\begin{array}{c}2.46(1.51-4.03) \\
1.89(1.18-3.01) \\
\text { Baseline }\end{array}$ & $<0.01$ \\
\hline Week of enrollment & $\begin{array}{l}\text { First week postpartum } \\
\text { Second week postpartum }\end{array}$ & $\begin{array}{c}0.49(0.32-0.74) \\
\text { Baseline }\end{array}$ & 0.03 \\
\hline
\end{tabular}

${ }^{1}$ Body condition score at day 5 postpartum was categorized (BCSC) as low $\leq 3.0$, moderate $>3.0$ or $<3.75$, or high $\geq 3.75$; calving score $(1=$ unassisted, $2=$ easy pull or slight problem, $3=$ moderate pull; and $4=$ hard pull) was used to determine dystocia (no: calving score $=1$ vs. yes: calving score $>1$ ); fever at the metritis diagnosis was categorized as yes $\geq 39.5^{\circ} \mathrm{C}$ vs. no $\leq 39.5^{\circ} \mathrm{C}$; milk slope up to 5 DIM and milk difference were categorized based on the percentile $(\mathrm{P})$ of milk production increased obtained from the linear regression (low $=\mathrm{P} 1-\mathrm{P} 25$; moderate $=\mathrm{P} 25-\mathrm{P} 75$, or high $>\mathrm{P} 75)$, milk production from the day before to the metritis diagnosis categoric (MilkVar); parity was categorized as primiparous vs. multiparous; retained of fetal membranes (RFM) were categorized as yes and no; vulvovaginal laceration (VLS) was categorized as $0=$ no laceration; $1=$ laceration $<2 \mathrm{~cm}$ at dorsal commissure or internal vaginal wall; $2=$ vulvovaginal laceration $>2 \mathrm{~cm}$; categorical VLS (VLC) was further divided into yes $(\geq 2 \mathrm{~cm})$ or no $(<2 \mathrm{~cm})$.

\section{Predictions of Metritis Cure from the Machine Learning Models}

The accuracies of the models for scenarios $1,2,3$, and 4 were $66,72,71$, and $70 \%$, respectively (Table 6 ).
The random forest classifier had the best performance across all 4 scenarios, whereas the best overall preprocessing pipeline was standard scaling for FS, KNN for MVI, and LOF for OD (Table 6). Scenario 2, which was trained using all features except for Vmilk and MilkVar,

Table 4. Univariate logistic regression model evaluating the association of several continuous variables with metritis cure

\begin{tabular}{lccc}
\hline Variable & Level & $\begin{array}{c}\text { Odds ratio } \\
(95 \% \mathrm{CI})\end{array}$ & $P$-value \\
\hline DIM at enrollment & 7 & Baseline & $<0.01$ \\
Milk production slope at 5 DIM & 8 & $1.17(1.06-1.28)$ & 0.01 \\
Milk difference $(\mathrm{kg})$ & 1.89 & Baseline & $<0.01$ \\
Number of lactations & 2.89 & $1.11(1.02-1.21)$ & 0.31 \\
Rectal temperature $\left({ }^{\circ} \mathrm{C}\right)$ & 0.57 & Baseline & 0.02 \\
\end{tabular}


Table 5. Outcomes from the final multivariable logistic regression model evaluating the association between cow-related factors and metritis cure

\begin{tabular}{llcc}
\hline Variable $^{1}$ & Level & $\begin{array}{c}\text { Odds ratio } \\
(95 \% \mathrm{CI})\end{array}$ & $P$-value \\
\hline Milk difference & Reduced $(\mathrm{n}=161)$ & $0.46(0.27-0.79)$ & $<0.01$ \\
& Moderate $(\mathrm{n}=151)$ & Baseline & \\
Temperature & High $(\mathrm{n}=103)$ & $0.92(0.48-1.78)$ & \\
& $39.40(\mathrm{n}=287)$ & Baseline & 0.08 \\
Treatment & $40.40(\mathrm{n}=263)$ & Baseline & $<0.01$ \\
VLC & CON $(\mathrm{n}=275)$ & $1.99(1.21-3.26)$ & $<0.01$ \\
CEF $(\mathrm{n}=275)$ & Baseline & \\
Week postpartum & Yes $(\mathrm{n}=403)$ & $1.91(1.17-3.14)$ & $<0.01$ \\
& No $(\mathrm{n}=147)$ & $0.49(0.29-0.81)$ & \\
\hline
\end{tabular}

${ }^{1}$ Vulvovaginal laceration (VLC) was categorized as $0=$ no laceration; $1=$ laceration $<2 \mathrm{~cm}$ at dorsal commissure or internal vaginal wall; $2=$ vulvovaginal laceration $>2 \mathrm{~cm}$; categorical vulvovaginal laceration score (VLC) was divided into yes $(\geq 2 \mathrm{~cm})$ laceration or no $(<2 \mathrm{~cm}$ laceration).

had the best performance based on the parsimonious point, which is the model that provides the simplest explanation that fits for best results when more than 1 option to choose is available. Scenario 2 had an F1 score of 0.81 , an accuracy of 0.72 , a PPV (precision) of 0.78 , an NPV of 0.50, a Se of 0.85 (recall), a Sp of 0.39, and false discovery rate of 0.22 .

\section{DISCUSSION}

Our results demonstrated that the cure for metritis is associated with cow-level and environmental factors, and that the use of machine learning algorithms needs to be optimized to become a feasible approach to identify cows with greater probability of metritis cure. The accuracy of classic multivariable model was $75 \%$, which was higher than the best machine learning scenario $(72 \%)$. However, considering the limited number of cows and variables used for the current study, more potential for improvement is expected with machine learning algorithms that are known to have an advantage handling large data sets and number of variables (Grzesiak, et al., 2010). Advancing predictive models for cure is a strategy that aligns with a worldwide effort to reduce the use of antibiotics in food animal systems, contributing to reducing the antimicrobial resistance problem, both in animals and humans (CDC, 2020).

In the current study, our goals were to investigate cow-level and environmental factors associated with metritis cure by integrating classic multivariable statistical models and machine learning algorithms, which were reported as an effective strategy for data mining and development of predictive tools for fertility and insemination outcomes (Shahinfar et al., 2012; Hempstalk et al., 2015), metabolic diseases (Xu et al., 2019), and mastitis (Hyde et al., 2020). An interesting

Table 6. Performance of machine learning algorithms for scenarios 1,2, 3, and 4; minmax feature scaling, iterative missing value imputation, local outlier factor (LOF) outlier detection, and random forest classifier were used to produce these results ${ }^{1}$

\begin{tabular}{|c|c|c|c|c|}
\hline \multirow[b]{2}{*}{ Item $^{2}$} & \multicolumn{4}{|c|}{ Scenario $^{3}$} \\
\hline & Scenario 1 & Scenario 2 & Scenario 3 & Scenario 4 \\
\hline Sensitivity & $0.81 \pm 0.03$ & $0.85 \pm 0.03$ & $0.85 \pm 0.04$ & $0.81 \pm 0.03$ \\
\hline Specificity & $0.26 \pm 0.07$ & $0.39 \pm 0.07$ & $0.35 \pm 0.3$ & $0.39 \pm 0.07$ \\
\hline PPV & $0.75 \pm 0.02$ & $0.78 \pm 0.02$ & $0.77 \pm 0.01$ & $0.78 \pm 0.02$ \\
\hline NPV & $0.33 \pm 0.06$ & $0.50 \pm 0.04$ & $0.49 \pm 0.09$ & $0.45 \pm 0.06$ \\
\hline Accuracy & $0.66 \pm 0.03$ & $0.72 \pm 0.02$ & $0.71 \pm 0.03$ & $0.70 \pm 0.03$ \\
\hline F1 score & $0.78 \pm 0.02$ & $0.81 \pm 0.02$ & $0.81 \pm 0.02$ & $0.79 \pm 0.02$ \\
\hline False detection rate & $0.25 \pm 0.02$ & $0.22 \pm 0.02$ & $0.23 \pm 0.01$ & $0.22 \pm 0.02$ \\
\hline
\end{tabular}

${ }^{1}$ All scenarios modeled included the final number of samples used for training the machine algorithms that was up-sampled to correct imbalance $(\mathrm{n}=794$, cured $=397$, and noncured $=397)$.

${ }^{2} \mathrm{PPV}=$ positive predictive value; $\mathrm{NPV}=$ negative predictive value; $\mathrm{F} 1$ score $=$ harmonic mean between precision and recall.

${ }^{3}$ All values $\pm \mathrm{SE}$. 


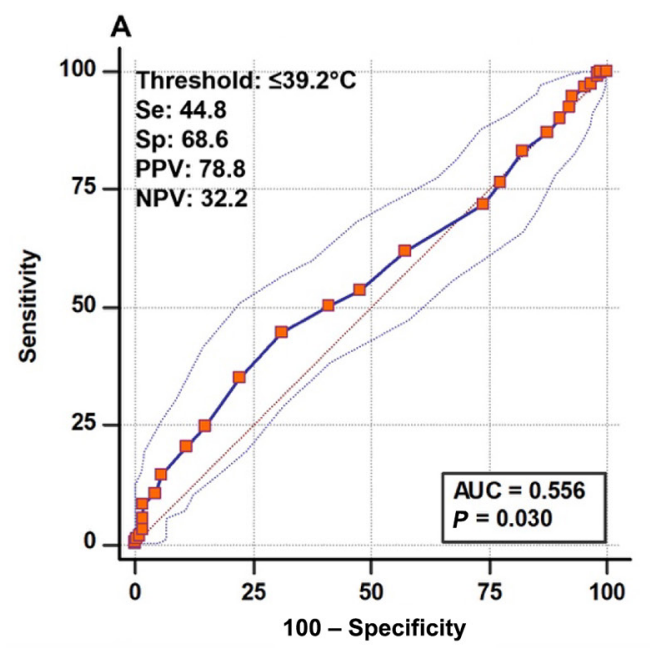

C

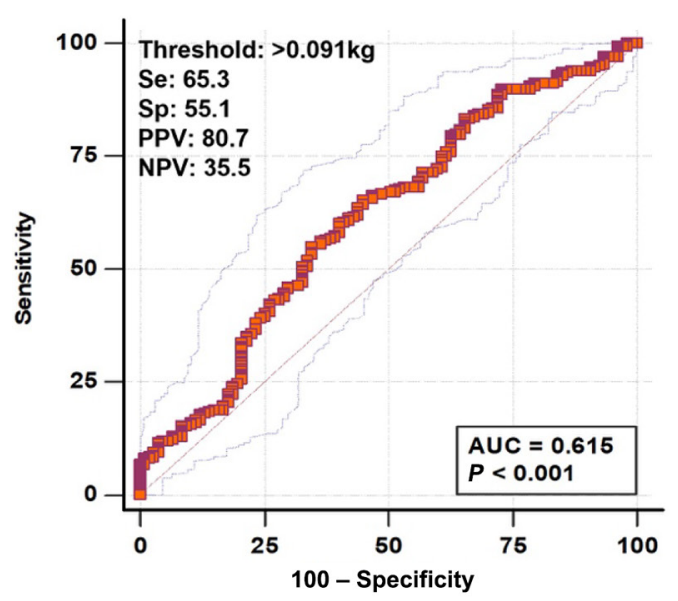

B

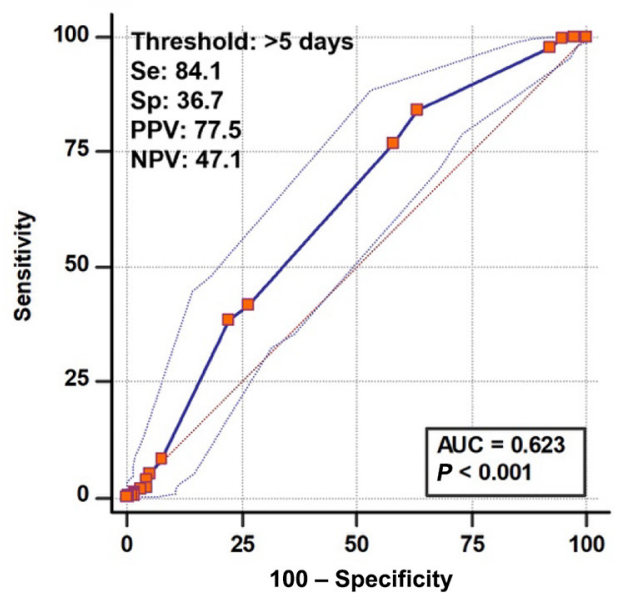

D

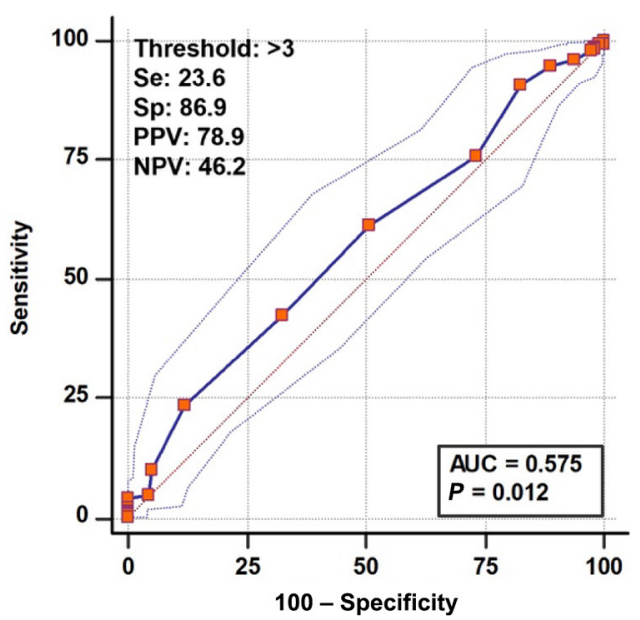

Figure 2. Optimal threshold values for rectal temperature at metritis diagnosis, DIM at diagnosis, milk difference from the day before diagnosis to the metritis diagnosis, and slope of milk increase up to 5 DIM. Outcome of 4 receiver operating characteristic curve analyses were performed to evaluate the optimum threshold of rectal temperature at metritis diagnosis (A), DIM at metritis diagnosis (B), milk increase (C), and milk slope associated with metritis cure (D). Se = sensitivity; $\mathrm{Sp}=$ specificity; PPV = positive predictive value; NPV = negative predictive value. $\mathrm{AUC}=$ area under the curve.

finding of our study was that calving-related disorders already known as risk factors for metritis such as parity, dystocia, BCS5, and RFM, were not included in the final multivariable logistic regression model because we did not detect an association with metritis cure up to $12 \mathrm{~d}$ after metritis diagnosis. It is unclear why these variables were not associated with cure by traditional logistic regression. Machado et al. (2020) found no association between parity and cure of metritis at $12 \mathrm{~d}$ after metritis diagnosis, consistent with our findings. Also, Lima et al. (2014) observed that parity was not associated with cure up to $12 \mathrm{~d}$ after enrollment. However, it was associated with cure within the first week after the initial diagnosis. Also, calving-related disorders such as dystocia, twin calves, and RFM were negatively associated with cure at $6 \mathrm{~d}$, but not $12 \mathrm{~d}$, after enrollment. Parity may be associated with an early cure, but the current study focused on the final assessment for the cure at $12 \mathrm{~d}$ after metritis diagnosis. That was the time point where the cure rate between treated and nontreated cows differed previously for data used herein (de Oliveira et al., 2020).

The vulvovaginal laceration was an important variable for metritis cure in both sets of analyses. Cows without vulvovaginal laceration had higher odds of curing of metritis than cows with vulvovaginal laceration. The relevance of vulvovaginal laceration and metritis cure in classical statistical and machine learning models suggests that this variable might be a more accurate predictor for metritis cure than calving-related 
disorders, which is a combination of RFM, dystocia, stillbirth, and twins. Each of the 4 components of calving-related disorders has been associated individually with metritis risk (Ghavi Hossein-Zadeh and Ardalan, 2011). Still, their association with metritis cure is less clear and, as suggested by the current study, is not consistently detected across different analytical methods. Both vulvovaginal laceration and calving-related disorders can increase tissue damage, and necrotic cells release damage-associated molecules or molecular patterns that increase the inflammatory response and delay healing (Healy et al., 2014). Perhaps the release of damage-associated molecules or molecular patterns is more consistent in cows with vulvovaginal laceration and helps explain why this variable was ubiquitously present in our models and analytical methods used in the current study.

Results from a pilot study suggested that leaving metritis cases that occurred within the first 5 DIM did not lead to negative outcomes (Sannmann et al., 2013), and only cows with fever at the metritis diagnosis would benefit of the antibiotic treatment. However, our results from the classic multivariable statistical and ROC curve analysis, in contrast with this recommendation, suggested that cows developing metritis in earlier lactation are less likely to cure than cows developing metritis after the first week postpartum. A possible reason for these results is that cows developing metritis earlier may have compromised immune response necessary to achieve uterine pathogen clearance, which can lead to increased uterine pathogen load and toxins production and lower efficacy of antibiotics treatment. Also, in the current study, cows with a rectal temperature $>39.4^{\circ} \mathrm{C}$ tended to have lower odds of being cured than cows with a rectal temperature $\leq 39.4^{\circ} \mathrm{C}$.

The current study results revealed that cows having reduced milk production from the day before diagnosis to the metritis diagnosis are less likely to cure than cows with moderate milk production. Machine learning and logistic regression analyses indicated that milk production is an important variable for the predictability of a metritis cure. In the present study, the machine learning analysis was trained on 4 different subsets of variables to create different scenarios. When milk production was not included in the analysis, machine learning predicted metritis cure with an accuracy of 0.66. However, when the different milk production measurements were added to the analysis in the other 3 scenarios, the metritis cure predictability increased with an accuracy greater than 0.70 . Traditional multivariable logistic regression analysis detected the slopes of milk production up to 5 DIM as the best variable to predict metritis cure, and the inclusion of only this variable generated a full model with the lowest AIC.
Machine learning analysis indicated that the model including slopes of milk production for 5, 7, and 9 DIM had the best accuracy to predict which cows cured of metritis, but the accuracy was still lower than the multivariate ROC analysis. The decrease in milk production with the onset of metritis can be attributed at least in part to a concomitant decrease in DMI associated with inflammation (Plata-Salamán et al., 1996; PérezBáez et al., 2019). Merenda et al. (2021) demonstrated that metritis profoundly affects inflammatory response, feeding, and rumination times compared with healthy cows. Immune activation markedly disrupts glucose homeostasis and is characterized by hypoglycemia and hyperlactemia (Filkins, 1978; McGuinness, 2005; Michaeli et al., 2012). The energy cost to keep the immune system activated under inflammation is very high (1,553 g for 720 min; Kvidera et al., 2017). Therefore, it is reasonable to surmise that cows experiencing metritis cure failure might exacerbate inflammatory response and activation of the immune system.

For most of the variables analyzed in the current study for an association or prediction of metritis cure, we could not see differences between cows treated and not treated for metritis. The only exception was a tendency for interaction between treatment and vulvovaginal laceration. The efficacy of ceftiofur to treat metritis is well established (Chenault et al., 2004; McLaughlin et al., 2012; de Oliveira et al., 2020). Cows without vulvovaginal laceration treated with ceftiofur had higher odds of curing of metritis than cows with vulvovaginal laceration, whereas a similar relationship was not found in the CON group. We speculate that animals with vulvovaginal laceration may have the severity of metritis increased, and the ceftiofur effectiveness to cure metritis might be diminished. Silva et al. (2021) reported in a recent study that cows with metritis that received an antibiotic treatment had lesser proportion of culling and similar mortality up to 300 DIM when compared with untreated cows. The data from Silva et al. (2021) suggest that increased culling rate may be related to the decreased milk production and reproductive performance experienced by nontreated metritic cows. Identifying cow-level and environmental factors leading to metritis cure might be a critical step to design a decision tree for selective treatment that goes beyond cure rate and positively affects fertility and milk yield performance.

The machine learning also selected all variables included in the multivariable logistic regression recommended system as a metritis cure predictor. Furthermore, machine learning analysis pointed out calvingrelated disorders as an important feature for metritis cure prediction, indicating that these variables are useful, and the development of a selective therapy for 
metritis using cow-related factors is a possibility. Using the AdaBoost algorithm, we were able to identify cowlevel and environmental factors that predict metritis cure with an accuracy of up to $72 \%$. Using the subset of variables selected by scenario 2 (all fixed features and milk production slope up to 5,7, and 9 DIM) in the machine learning models, $72 \%$ accuracy was achieved to determine whether the cow will cure or not from metritis. Ideally, an algorithm to be used to select cows to optimize cure without compromising animal welfare needs to have high sensitivity, high PPV, and moderate specificity. Our machine learning models had sensitivities ranging from 0.81 to 0.85 and PPV ranging from 0.75 to 0.78 , which suggest a good prediction toward judicious antimicrobial use. On the other hand, the specificities ranged from 0.26 to 0.39 , suggesting that from an animal welfare standpoint, algorithms need to be improved. A major limitation of the present study was that the data used for the analysis was only from 3 herds. Additionally, the present model was not validated using external data from other herds across the United States or with differences in metritis prevalence. Validation of the results of the current study could be done by applying the algorithm in a further cohort field study in which cows would be selected to receive or not treatment based on the algorithm. Nonetheless, our work demonstrated that it may be possible to identify cows with greater probability of cure. Further studies are necessary to improve the prediction ability of the model and may be achieved by including other markers of inflammation and immunomodulation as well as other cow-related factors such as genomics, rumination, activity level, and DMI.

\section{CONCLUSIONS}

Overall, our multivariable logistic regression and ROC analysis indicated that cows developing metritis at $>7$ DIM, with increase in milk production, and with a rectal temperature $\leq 39.40^{\circ} \mathrm{C}$ have increased likelihood of cure of metritis with an accuracy of $75 \%$ and Se of $98 \%$, which were both slightly higher than the values reported in our machine learning algorithm scenarios. Our findings from machine learning showed that in addition to these variables, considering calving-related disorders (dystocia, RFM, calf sex, twins, and stillbirth), season, the month of metritis event, and milk production slope up to 5,7 , and 9 DIM were helpful to predict whether the cow will cure or not from metritis. There is tremendous pressure on agriculture to improve the judicious use of antibiotics, and metritis has been shown to be one of the major drivers of antimicrobial drug usage in lactating cows, underscoring the impor- tance of predicting metritis cure. The unique capacity of machine learning algorithm to handle large data sets and number of variables may contribute to advance the development of selective treatment for metritis, potentially reducing the dissemination of antimicrobial resistance, concurrent with lower disease cost and improved animal welfare.

\section{ACKNOWLEDGMENTS}

The data used for the analysis performed in the current study was generated with the support of a grant from the USDA-National Institute of Food and Agriculture (NIFA)-Agriculture and Food Research Initiative (AFRI) program (Washington, DC; accession number: 1008863). Data analysis resources were generated with the support of the 2019-2020 VMTH Resident Research Grant at the School of Veterinary Medicine, University of California, Davis. The authors have not stated any conflicts of interest.

\section{REFERENCES}

Benzaquen, M. E., C. A. Risco, L. F. Archbald, P. Melendez, M.-J. Thatcher, and W. W. Thatcher. 2007. Rectal temperature, calving-related factors, and the incidence of puerperal metritis in postpartum dairy cows. J. Dairy Sci. 90:2804-2814. https://doi.org/10 .3168/jds.2006-482.

Breiman, L. 2001. Random forests. Mach. Learn. 45:5-32. https://doi .org/10.1023/A:1010933404324

Breunig, M., H.-S. Kriegel, R. T. Ng, and J. Sander. 2000. LOF: Identifying density-based local outliers. Pages 93-104 in SIGMOD International Conference on Management of Data. SIGMOD International Conference on Management of Data and Symposium on Principles of Database Systems.

CDC. 2020. US national strategy for combating antibiotic-resistant bacteria (national action plan). Accessed Feb. 2, 2021. https:// www.cdc.gov/drugresistance/us-activities/national-action-plan .html

Chenault, J. R., J. F. McAllister, S. Theodore Chester, K. J. Dame, F. M. Kausche, and E. J. Robb. 2004. Efficacy of ceftiofur hydrochloride sterile suspension administered parenterally for the treatment of acute postpartum metritis in dairy cows. J. Am. Vet. Med. Assoc. 224:1634-1639. https://doi.org/10.2460/javma.2004.224.1634. de Oliveira, E. B., F. Cunha, R. Daetz, C. C. Figueiredo, R. C. Chebel, J. E. P. Santos, C. A. Risco, K. C. Jeong, V. S. Machado, and K. N. Galvão. 2020. Using chitosan microparticles to treat metritis in lactating dairy cows. J. Dairy Sci. 103:7377-7391. https://doi.org/ 10.3168/jds.2019-18028.

de Oliveira, E. B., F. C. Ferreira, K. N. Galvão, J. Youn, I. Tagkopoulos, N. Silva-del-Rio, R. V. V. Pereira, V. S. Machado, and F. S. Lima. 2021. Integration of statistical inferences and machine learning algorithms for prediction of metritis cure in dairy cows. Supplemental materials. Accessed Sept. 7, 2021. https://figshare .com/s/fa21c3120f945d38ff36.

Dohoo, I., W. Martin, and H. Stryhn. 2009. Veterinary Epidemiological Research. VER Inc.

Domingos, P., and M. Pazzani. 1997. On the optimality of the simple Bayesian classifier under zero-one loss. Mach. Learn. 29:103-130. https://doi.org/10.1023/A:1007413511361.

Dubuc, J., T. F. Duffield, K. E. Leslie, J. S. Walton, and S. J. LeBlanc. 2010. Risk factors for postpartum uterine diseases in dairy cows. J. Dairy Sci. 93:5764-5771. https://doi.org/10.3168/jds.2010-3429. 
Fair, R. J., and Y. Tor. 2014. Antibiotics and bacterial resistance in the 21st century. Perspect. Medicin. Chem. 6:25-64. https://doi .org/10.4137/PMC.S14459.

FDA. 2020. FDA-TRACK: Progress on FDA's support of antimicrobial stewardship in veterinary settings. Accessed Feb. 5, 2020 https://www.fda.gov/about-fda/fda-track-agency-wide-program -performance/fda-track-progress-fdas-support-antimicrobial -stewardship-veterinary-settings.

Ferguson, J. D., D. T. Galligan, and N. Thomsen. 1994. Principal descriptors of body condition in Holstein dairy cattle. J. Dairy Sci. 77:2695-2703.

Filkins, J. P. 1978. Phases of glucose dyshomeostasis in endotoxicosis. Circ. Shock 5:347-355.

Ghavi Hossein-Zadeh, N., and M. Ardalan. 2011. Cow-specific risk factors for retained placenta, metritis and clinical mastitis in Holstein cows. Vet. Res. Commun. 35:345-354.

Grzesiak, W., D. Zaborski, P. Sablik, A. Zukiewicz, A. Dybus, and I. Szatkowska. 2010. Detection of cows with insemination problems using selected classification models. Comput. Electron. Agric. 74:265-273. https://doi.org/10.1016/j.compag.2010.09.001.

Healy, L. L., J. Cronin, and I. Sheldon. 2014. Endometrial cells sense and react to tissue damage during infection of the bovine endometrium via interleukin 1. Sci. Rep. 4:7060. https://doi.org/10.1038/ srep07060.

Hempstalk, K., S. McParland, and D. Berry. 2015. Machine learning algorithms for the prediction of conception success to a given insemination in lactating dairy cows. J. Dairy Sci. 98:5262-5273. https://doi.org/10.3168/jds.2014-8984.

Ho, T. K. 1995. Random decision forest. Pages 278-282 in Proc. 3rd International Conference on Document Analysis and Recognition. IEEE.

Hu, L. Y., M. W. Huang, S. W. Ke, and C. F. Tsai. 2016a. The distance function effect on k-nearest neighbor classification for medical datasets. Springerplus 5:1304. https://doi.org/10.1186/s40064 $-016-2941-7$.

Hu, Y., T. Hase, H. P. Li, S. Prabhakar, H. Kitano, S. K. Ng, S. Ghosh, and L. J. Wee. 2016b. A machine learning approach for the identification of key markers involved in brain development from single-cell transcriptomic data. BMC Genomics 17(Suppl. 13):1025. https://doi.org/10.1186/s12864-016-3317-7.

Hyde, R. M., P. M. Down, A. J. Bradley, J. E. Breen, C. Hudson, K. A. Leach, and M. J. Green. 2020. Automated prediction of mastitis infection patterns in dairy herds using machine learning. Sci. Rep. 10:4289. https://doi.org/10.1038/s41598-020-61126-8.

Kossaibati, M. A., and R. J. Esslemont. 1997. The costs of production diseases in dairy herds in England. Vet. J. 154:41-51. https://doi .org/10.1016/S1090-0233(05)80007-3.

Kvidera, S. K., E. A. Horst, M. Abuajamieh, E. J. Mayorga, M. V. Sanz Fernandez, and L. H. Baumgard. 2017. Glucose requirements of an activated immune system in lactating Holstein cows. J. Dairy Sci. 100:2360-2374. https://doi.org/10.3168/jds.2016-12001.

LeBlanc, S. J., T. F. Duffield, K. E. Leslie, K. G. Bateman, G. P. Keefe, J. S. Walton, and W. H. Johnson. 2002. Defining and diagnosing postpartum clinical endometritis and its impact on reproductive performance in dairy cows. J. Dairy Sci. 85:2223-2236. https://doi.org/10.3168/jds.S0022-0302(02)74302-6.

Lima, F. S., A. Vieira-Neto, G. S. F. M. Vasconcellos, R. D. Mingoti, E. Karakaya, E. Solé, R. S. Bisinotto, N. Martinez, C. A. Risco, K. N. Galvão, and J. E. P. Santos. 2014. Efficacy of ampicillin trihydrate or ceftiofur hydrochloride for treatment of metritis and subsequent fertility in dairy cows. J. Dairy Sci. 97:5401-5414. https:/ /doi.org/10.3168/jds.2013-7569.

Liu, F. T., K. M. Ting, and Z. Zhou. 2008. Isolation Forest. Pages 413-422 in Eighth IEEE International Conference on Data Mining. IEEE Computer Society.

Lu, J., D. Wu, M. Mao, W. Wang, and G. Zhang. 2015. Recommender system application developments: A survey. Decis. Support Syst. 74:12-32. https://doi.org/10.1016/j.dss.2015.03.008.

Machado, V. S., M. L. Celestino, E. B. Oliveira, F. S. Lima, M. A. Ballou, and K. N. Galvão. 2020. The association of cow-related factors assessed at metritis diagnosis with metritis cure risk, reproductive performance, milk yield, and culling for untreated and ceftiofurtreated dairy cows. J. Dairy Sci. 103:9261-9276. https://doi.org/ 10.3168/jds.2020-18643.

Markusfeld, O. 1987. Periparturient traits in seven high dairy herds. Incidence rates, association with parity, and interrelationships among traits. J. Dairy Sci. 70:158-166. https://doi.org/10.3168/ jds.S0022-0302(87)79990-1.

McGuinness, O. P. 2005. Defective glucose homeostasis during infection. Annu. Rev. Nutr. 25:9-35. https://doi.org/10.1146/annurey .nutr.24.012003.132159.

McLaughlin, C. L., E. Stanisiewski, M. J. Lucas, C. P. Cornell, J. Watkins, L. Bryson, J. K. S. Tena, J. Hallberg, and J. R. Chenault 2012. Evaluation of two doses of ceftiofur crystalline free acid sterile suspension for treatment of metritis in lactating dairy cows. J Dairy Sci. 95:4363-4371. https://doi.org/10.3168/jds.2011-5111.

Merenda, V. R., D. Lezier, A. Odetti, C. C. Figueiredo, C. A. Risco, R. S. Bisinotto, and R. C. Chebel. 2021. Effects of metritis treatment strategies on health, behavior, reproductive, and productive responses of Holstein cows. J. Dairy Sci. 104:2056-2073. https:// doi.org/10.3168/jds.2020-19076.

Michaeli, B., A. Martinez, J. P. Revelly, M. C. Cayeux, R. L. Chioléro, L. Tappy, and M. M. Berger. 2012. Effects of endotoxin on lactate metabolism in humans. Crit. Care 16:R139. https://doi.org/ $10.1186 /$ cc11444.

Mitchell, T. M. 1997. Artificial neural networks. Pages 111-112 in Machine Learning. McGraw-Hill International Edition.

Morra, J. H., Z. Tu, L. G. Apostolova, A. E. Green, A. W. Toga, and P. M. Thompson. 2010. Comparison of AdaBoost and support vector machines for detecting Alzheimer's disease through automated hippocampal segmentation. IEEE Trans. Med. Imaging 29:30-43. https://doi.org/10.1109/TMI.2009.2021941.

Pérez-Báez, J., C. A. Risco, R. C. Chebel, G. C. Gomes, L. F. Greco, S. Tao, I. M. Thompson, B. C. do Amaral, M. G. Zenobi, N. Martinez, C. R. Staples, G. E. Dahl, J. A. Hernández, J. E. P. Santos, and K. N. Galvão. 2019. Association of dry matter intake and energy balance prepartum and post-partum with health disorders post-partum: Part I. Calving disorders and metritis. J. Dairy Sci. 102:9138-9150. https://doi.org/10.3168/jds.2018-15878.

Plata-Salamán, C. R., G. Sonti, J. P. Borkoski, C. D. Wilson, and J. M. H. French-Mullen. 1996. Anorexia induced by chronic central administration of cytokines at estimated 583 pathophysiological concentrations. Physiol. Behav. 60:867-875. https://doi.org/10 .1016/0031-9384(96)00148-5.

Sannmann, I., O. Burfeind, R. Voigtsberger, and W. Heuwieser. 2013. Comparison of two monitoring and treatment strategies for cows with acute puerperal metritis. Theriogenology 79:961-969. https:/ /doi.org/10.1016/j.theriogenology.2013.01.016.

Shahinfar, S., H. Mehrabani-Yeganeh, C. Lucas, A. Kalhor, M. Kazemian, and K. A. Weigel. 2012. Prediction of breeding values for dairy cattle using artificial neural networks and neuro-fuzzy systems. Comput. Math. Methods Med. 2012:127130. https://doi .org/10.1155/2012/127130.

Sheldon, I. M., M. Bushnell, J. Montgomery, and A. N. Rycroft. 2004 Minimum inhibitory concentrations of some anti-microbial drugs against bacteria causing uterine infections in cattle. Vet. Rec. 15:383-387.

Sheldon, I. M., J. Cronin, L. Goetze, G. Donofrio, and H. J. Schuberth. 2009. Defining postpartum uterine disease and the mechanisms of infection and immunity in the female reproductive tract in cattle. Biol. Reprod. 81:1025-1032. https://doi.org/10.1095/biolreprod 109.077370

Sheldon, I. M., G. S. Lewis, S. LeBlanc, and R. O. Gilbert. 2006. Defining post-partum uterine disease in cattle. Theriogenology 65:1516-1530. https://doi.org/10.1016/j.theriogenology.2005.08 .021 .

Silva, T. V., E. B. de Oliveira, J. Pérez-Báez, C. A. Risco, R. C. Chebel, F. Cunha, R. Daetz, J. E. P. Santos, F. S. Lima, K. C. Jeong, and K. N. Galvão. 2021. Economic comparison between ceftiofur-treated and nontreated dairy cows with metritis. J. Dairy Sci. 104:8918-8930. https://doi.org/10.3168/jds.2020-19430. 
Stekhoven, D. J., and P. Bühlmann. 2012. MissForest non-parametric missing value imputation for mixed-type data. Bioinformatics 28:112-118. https://doi.org/10.1093/bioinformatics/btr597.

Stojkov, J., M. A. G. Von Keyserlingk, J. N. Marchant-Forde, and D. M. Weary. 2015. Assessment of visceral pain associated with metritis in dairy cows. J. Dairy Sci. 98:5352-5361. https://doi.org/10 $.3168 /$ jds.2014-9296.

Troyanskaya, O., M. Cantor, G. Sherlock, P. Brown, T. Hastie, R. Tibshirani, D. Botstein, and R. B. Altman. 2001. Missing value estimation methods for DNA microarrays. Bioinformatics 17:520-525. https://doi.org/10.1093/bioinformatics/17.6.520.

Valdés, M. G., I. Galván-Femenía, V. R. Ripoll, X. Duran, J. Yokota, R. Gavaldà, X. Rafael-Palou, and R. de Cid. 2018. Pipeline design to identify key features and classify the chemotherapy response on lung cancer patients using large-scale genetic data. BMC Syst. Biol. 12(Suppl. 5):97. https://doi.org/10.1186/s12918-018-0615-5.

van Buuren, S. and G. O. Karin. 2010. mice: Multivariate imputation by chained equations in R. J. Stat. Softw. 45: 1-68.

Vieira-Neto, A., F. S. Lima, J. E. P. Santos, R. D. Mingoti, G. S. Vasconcellos, C. A. Risco, and K. N. Galvao. 2016. Vulvovaginal laceration as a risk factor for uterine disease in post-partum dairy cows. J. Dairy Sci. 99:4629-4637. https://doi.org/10.3168/jds.2016 -10872 .

Witten, I. H., E. Frank, and M. A. Hall. 2011. Data Mining: Practical Machine Learning Tools and Techniques. Morgan Kaufmann.
Xu, W., A. T. M. van Knegsel, J. J. M. Vervoort, R. M. Bruckmaier, R. J. van Hoeij, B. Kemp, and E. Saccenti. 2019. Prediction of metabolic status of dairy cows in early lactation with on-farm cow data and machine learning algorithms. J. Dairy Sci. 102:1018610201. https://doi.org/10.3168/jds.2018-15791.

Zafeiris, D., S. Rutella, and G. R. Ball. 2018. An artificial neural network integrated pipeline for biomarker discovery using Alzheimer's disease as a case study. Comput. Struct. Biotechnol. J. 16:77-87. https://doi.org/10.1016/j.csbj.2018.02.001.

\section{ORCIDS}

E. B. de Oliveira @ https://orcid.org/0000-0002-0434-8501

F. C. Ferreira @ https://orcid.org/0000-0003-2387-2269

K. N. Galvão ๑ https://orcid.org/0000-0002-6683-4476

J. Youn (1) https://orcid.org/0000-0002-5925-0261

N. Silva-del-Rio () https://orcid.org/0000-0002-2826-6797

R. V. V. Pereira @ https://orcid.org/0000-0003-2028-8761

V. S. Machado ํ https://orcid.org/0000-0003-1513-8216

F. S. Lima @ https://orcid.org/0000-0001-8377-6469 